\title{
Prethermalization and glassiness in the bosonic Hubbard model
}

\author{
I. Salazar Landea and N. Nessi \\ Instituto de Física La Plata (IFLP) - CONICET and Departamento de Física, Universidad Nacional de La Plata, \\ CC 67, 1900 La Plata, Argentina
}

(Received 9 March 2015; published 2 June 2015)

\begin{abstract}
We investigate the nonequilibrium dynamics of the bosonic Hubbard model starting from inhomogeneous superfluid or Mott insulator initial states using the truncated Wigner approximation (TWA). We find that the relaxation of the system develops in two steps for sufficiently large interaction strengths: after a fast relaxation the system gets caught in metastable prethermalized states that precede the true equilibrium state. We find that the lifetime of these prethermalized states increases by several orders of magnitude as we increase the on-site interaction strength beyond a threshold value. We show that the emergence of long-lived metastable states in the quantum dynamics is associated with an ergodic (active) to nonergodic (inactive) dynamical phase transition in the ensemble of classical trajectories that contribute to the semiclassical limit. This dynamical phase transition, which is very similar to that found in different classical models of glasses, is closely related to the dynamic heterogeneity of the classical relaxation.
\end{abstract}

DOI: 10.1103/PhysRevA.91.063601

PACS number(s): 03.75.Kk, 05.70.Ln, 67.85.Hj

\section{INTRODUCTION}

The nonequilibrium dynamics of closed quantum systems is nowadays a very active field of research [1]. The motivations are manifold. On one hand, the determination of the requirements that the initial state and the nonequilibrium protocol have to fulfill to ensure that a closed quantum system will reach asymptotically a state of thermal equilibrium constitutes a key issue in the study of the foundations of statistical mechanics. On the other hand, with the advent of cold atomic systems the manipulation of nearly perfectly isolated quantum systems with large coherence times has become experimentally feasible [2]. There are several theoretical and experimental examples of systems that starting from nonequilibrium conditions reach, on accessible time scales, a state that is compatible with thermal equilibrium [3-6], at least at the level of simple correlation functions. However, situations in which thermal equilibration is elusive are clearly of particular interest. For integrable systems, thermal equilibration is not expected since the large number of constants of motion prevent the system from erasing memory of the initial conditions. However, there are examples of systems that are not integrable (but are close to an integrable point) for which thermal equilibration is not observed on the accessible time scales. Maybe the first experimental example of a (nonintegrable) closed quantum many-body system failing to reach thermal equilibrium on accessible time scales was provided by the famous experiment of Kinoshita et al. [7].

The phenomenon of prethermalization [8-15] is one possible explanation for the apparent lack of thermalization in nearly integrable systems. Indeed, in certain situations it can be shown that the relaxation of systems close to an integrable point may proceed in two steps. The first step is a fast relaxation whose main mechanism is dephasing between some quasifree modes of the system [12]. Close to an integrable point, where other relaxation mechanisms such as inelastic collisions are extremely inefficient, such dephasing relaxation often gives rise to a highly nonthermal metastable state. Afterward, the remaining relaxation channels become relevant and the prethermal metastable state starts to decay to the final state of the system $[9,10,16]$. One of the most characteristic phenomenons associated with prethermalization is the fact that certain observables reach its final, thermal value in the short time scales of the dephasing relaxation. The kinetic energy of the system is the most prominent example of such behavior [8]. Prethermalization has been observed in experiments with ultracold bosonic gases $[13,17]$. We must also mention that finite-size systems close to an integrable point may also show problems in thermalizing due to the breakdown of the eigenstate thermalization hypothesis (ETH) [18], a phenomenon that is not related to prethermalization but whose manifestations may be blended with it in the dynamics of small systems.

Prethermalization may be behind the apparent breakdown of thermalization in the Bose-Hubbard model, which, for strong interactions, is close to the atomic limit integrable point. Starting with the pioneering work of Kollath et al. [19], where using density matrix renormalization group (DMRG) techniques it was shown that for sufficiently strong interactions few-body correlation functions reached a quasistationary state that was not compatible with thermal equilibrium, several studies have focused on this system [20-22]. In Ref. [21] it was shown that for strong interactions the system gets trapped in long-lived inhomogeneous metastable states. Such kinetic arrest was shown [21] to be associated with a dynamical localization in the many-body Hilbert space. The BoseHubbard model is also one of the most extensively realized models in cold-atom experiments $[2,23,24]$. In particular, experiments with inhomogeneous Bose-Einstein condensates also demonstrate pronounced time-scale separation and slow thermalization for strong interactions [25-27].

On the other hand, since the seminal work of Srednicki about the ETH [28], semiclassical considerations have been very important in our understanding of quantum thermalization. Recently Cosme et al. [29] made a contribution in this line through the truncated Wigner approximation (TWA) applied to a two-site multiband bosonic Hubbard model. Since the TWA allows us to consider the first-order quantum corrections 
to the classical action [30] the connection between quantum physics and its classical limit becomes explicit. In particular, it has been checked in Ref. [29] that the thermalization of the quantum system comes along with the ergodicity of classical trajectories. Also along these lines, of particular relevance to the study presented here are the works in Refs. [31,32] where the breakdown of thermalization for strong interactions was linked with the chaotic properties of the mean-field limit. More precisely, it was shown that the Lyapunov exponent of the classical trajectories associated with the solutions of the mean-field equations is suppressed for strong interactions.

The appearance of long-lived prethermalized states in the relaxation of isolated quantum systems is phenomenologically very similar to the physics of classical glassy systems such as spin glasses [33], atomistic glass formers [34,35], and kinetically constrained models [36]. Such systems exhibits a typical two-step relaxation: after a fast inertial regime (analogous to the dephasing regime) they get caught in metastable states whose lifetime can be astronomical in certain parameter regions, for example, for sufficiently low temperature or high density in the case of atomic models. One of the most distinctive characteristics of glassy relaxation is the appearance of dynamical heterogeneity [36], i.e., the fact that the relaxation is spatially nonhomogeneous: fast and slow regions are clustered together. One of the most appealing theories to explain such a feature is that of a dynamical phase transition between active and inactive phases taking place in the ensemble of possible histories that the system can follow in the relaxation [35,37-39].

In this work we revisit the problem of the apparent breakdown of thermalization in the bosonic Hubbard model from the semiclassical perspective provided by the TWA. For large occupation numbers, this approach allows us to access the dynamics of the quantum system performing weighted averages over ensembles of classical (mean field) trajectories. We explicitly show that prethermalized states can have a huge lifetime that grows exponentially while increasing the coupling strength, which prevents the observation of thermal equilibration on any reasonable time scale. Moreover, we find that the emergence of long-lived prethermalized states is closely related to the appearance of nonergodic, glassy features in the mean-field trajectories associated with the quantum dynamics. More specifically, we find that the ensemble of mean-field trajectories undergo a dynamical phase transition between an active and an inactive phase, completely analogous to that observed in classical models of glasses. We find that in the present case this transition is also intimately correlated with a remarkable phenomenon present in the mean-field trajectories, dynamical heterogeneity. We shall go deeper into these issues in the remaining sections of the paper, which are organized as follows. In Sec. II we describe the model, the initial conditions, and the semiclassical approximation used to study the quantum dynamics and show the results for the quantum dynamics, while in Sec. III we thoroughly investigate the properties of the ensemble of mean-field trajectories that contribute to the quantum dynamics and show that they show clear signatures of glassiness as we increase the strength of the interaction. In Sec. IV we briefly summarize our results.

\section{QUANTUM DYNAMICS}

Let us consider the one-dimensional bosonic Hubbard model with Hamiltonian $H=H_{0}+H_{\text {int }}$ :

$$
\begin{aligned}
H_{0} & =-J \sum_{j=1}^{L}\left(a_{j}^{\dagger} a_{j+1}+a_{j+1}^{\dagger} a_{j}\right), \\
H_{\mathrm{int}} & =U \sum_{j=1}^{L} n_{j} n_{j}
\end{aligned}
$$

where $L$ is the number of sites in the chain, the $a$ 's are canonical bosonic operators, and $n_{j}=a_{j}^{\dagger} a_{j}$. We shall consider periodic boundary conditions, $a_{L+m}^{\#}=a_{m}^{\#}$. We shall analyze the dynamics generated by Eq. (1) starting from an inhomogeneous initial state described by a density matrix $\rho_{0}$, which we left unspecified until the next section. In particular, we shall focus on the relaxation of the density profile $\left\langle\hat{n}_{j}(t)\right\rangle=\operatorname{Tr}\left[\rho_{0} e^{i H t} \hat{n}_{j} e^{-i H t}\right]$, where we have set $\hbar=1$. The thermal density profile is given by $\left\langle\hat{n}_{j}\right\rangle_{\text {th }}=N_{a} / L=N$ for all $j$, where $N_{a}$ is the total number of bosons.

When the average number of atoms per site $N$ is large the dynamics of the Bose-Hubbard model can be studied using the TWA [30,40,41]. To calculate the time dependence of expectation values within the TWA we have to consider the solution of the classical equations of motion associated with the quantum Hamiltonian (1). These are the standard lattice Gross-Pitaevskii equations [30,40,41],

$$
i \frac{d \psi_{j}(t)}{d t}=-J\left[\psi_{j+1}(t)+\psi_{j-1}(t)\right]+2 U \psi_{j}(t)\left|\psi_{j}(t)\right|^{2},
$$

where the classical fields are normalized to the total number of particles $\sum_{j=1}^{L}\left|\psi_{j}(t)\right|^{2}=N_{a}$. Then the expectation value of any given operator $\hat{\Omega}$ at time $t,\langle\hat{\Omega}(t)\rangle$, can be calculated averaging the corresponding classical observable $\Omega$ over an ensemble of initial conditions weighted according to the Wigner transform of the initial density matrix $\rho_{0}$ :

$$
\langle\hat{\Omega}(t)\rangle=\int d \psi_{0}^{*} d \psi_{0} p\left(\psi_{0}, \psi_{0}^{*}\right) \Omega\left(\psi(t), \psi^{*}(t)\right),
$$

where $p$ is defined as

$$
\begin{aligned}
p\left(\psi_{0}, \psi_{0}^{*}\right)= & \int d \eta_{0}^{*} d \eta_{0}\left\langle\psi_{0}-\frac{\eta_{0}}{2}\left|\rho_{0}\right| \psi_{0}+\frac{\eta_{0}}{2}\right\rangle \\
& \times e^{-\left|\psi_{0}\right|^{2}-\frac{1}{4}\left|\eta_{0}\right|^{2}} e^{\frac{1}{2}\left(\eta_{0}^{*} \psi_{0}-\eta_{0} \psi_{0}^{*}\right)}
\end{aligned}
$$

where $\left|\psi_{0} \pm \frac{\eta_{0}}{2}\right\rangle$ is a coherent state with eigenvalue $\psi_{0} \pm \frac{\eta_{0}}{2}$ with respect to the annihilation operator $a$. To lighten the notation we have omitted the site index $j$. The measure is

$$
d \psi_{0}^{*} d \psi_{0}=\prod_{j=1}^{L} d \psi_{j}^{*}(0) d \psi_{j}(0)
$$

The correspondence between classical and quantum observables can be formulated most easily using the coherent 
state Bopp representation, which makes the assignments

$$
\begin{gathered}
\hat{a}^{\dagger} \rightarrow \psi^{*}-\frac{1}{2} \frac{\partial}{\partial \psi}, \\
\hat{a} \rightarrow \psi+\frac{1}{2} \frac{\partial}{\partial \psi^{*}},
\end{gathered}
$$

so that

$$
\hat{n}_{j} \rightarrow n_{j}=\left|\psi_{j}\right|^{2}-\frac{1}{2} .
$$

The TWA is the leading-order approximation in an expansion in a small parameter that measures deviations from classicality [30]. In the particular case of interacting bosons this parameter is $1 / N$, the inverse of average number of bosons per site. Thus, the most basic requirement to ensure the accuracy of the TWA is to work in the regime of high density [41,42], to which we shall circumscribe in the following. The TWA is a controlled approximation in the sense that it is possible to calculate higher order corrections that take the form of stochastic perturbations to the classical trajectories [30]. However, as with any approximation, be it controlled or not, there are certain parameter regimes in which it works better than others. To define when we expect this approximation to be accurate we may introduce the nonlinearity parameter $\lambda=\frac{U N}{J}$, which is the ratio between the typical potential energy per site and the typical kinetic energy per site [32]. When the interactions are strong enough, $\lambda \sim N^{2}$, the system (in equilibrium) undergoes a quantum phase transition to a Mott insulating state. In the vicinity of the transition, quantum fluctuations become large and we cannot expect the TWA to work well in this case, i.e., second-order corrections become important in that regime. However, there is a wide regime $\lambda \lesssim N^{2}(U / J \lesssim N)$ where the ground state of the system is a superfluid (weakly or strongly interacting depending on whether $\lambda<1$ or $\lambda>1$ respectively) where we can expect the TWA to be a good approximation to the dynamics. We shall work in that regime.

In parallel, the choice of the initial condition is also relevant for the accuracy of the results. We shall work with two types of initial states: coherent (superfluid) and Fock (Mott insulator). The coherent state represents the initial condition that is closest to a perfectly defined classical initial condition and therefore we can expect the TWA to accurately capture the dynamics. In particular, the Wigner function is

$$
p_{C}\left(\psi_{0}, \psi_{0}^{*}\right)=\prod_{j=1}^{L} \frac{2}{\pi} e^{-2\left|\psi_{j}-\sqrt{N_{j}}\right|^{2}},
$$

where $N_{j}=\left\langle\hat{n}_{j}(0)\right\rangle$. The Wigner function is a true probability distribution and can be sampled efficiently as $\psi_{j}=\sqrt{N_{j}}+$ $\frac{1}{2}\left(\eta_{1}+i \eta_{2}\right)$, where $\eta_{1}$ and $\eta_{2}$ are two real Gaussian random variables with correlations $\overline{\eta_{j}}=0$ and $\overline{\eta_{j} \eta_{k}}=\delta_{j k}[29,43]$.

The Fock initial state is the least classical initial state in the sense that the initial phases are completely unspecified. The Wigner function in this case is

$$
p_{F}\left(\psi_{0}, \psi_{0}^{*}\right)=\prod_{j=1}^{L} 2 e^{-2\left|\psi_{j}\right|^{2}} L_{N_{j}}\left(4\left|\psi_{j}\right|^{2}\right),
$$

where $L_{N}(x)$ is the $N$ th-order Laguerre polynomial. Unfortunately, this $p$ generically is not definite positive and for large $N$ has a highly oscillatory behavior. This fact makes convergence much slower when performing the integration over initial conditions. To bypass this issue we shall approximate this Wigner function by products of true distributions reproducing the first moments of the Laguerre polynomials. The simplest way to do this is just to reproduce the first moment by replacing each factor $p_{N_{j}}\left(\psi_{j}^{*}, \psi_{j}\right)$ by a Dirac delta function $\delta\left(\left|\psi_{j}\right|^{2}-\right.$ $\frac{1}{2}-N_{j}$ ) and averaging over random phases $[29,43,44]$. The next step would be considering a distribution with two moments, i.e., the Gaussian $\frac{2}{\sqrt{2 \pi}} e^{-2\left(\left|\psi_{j}\right|^{2}-\frac{1}{2}-N_{j}\right)^{2}}$. We will refer to these two approximations as the delta and Gaussian Wigner functions $p_{\delta}$ and $p_{g}$ respectively. We have checked that in the particular cases that we investigated, both approaches give very similar results. We shall therefore use the $\delta$-function approximation due to computational convenience. For the Fock initial state it was found that, in the worst case, the TWA results quantitatively deviate from available exact solutions for times larger than $t_{c}=J / U$ [40]. However, the predictions of the TWA remain qualitatively valid for larger times.

We shall now proceed to investigate the quantum dynamics of the density profile. We shall choose the coherent or Fock initial states in such a way that the initial density profile is $(20,0,20,0, \ldots):$ an alternation of empty and highly occupied sites. With this election $N=10$. The system is on a ring of size $L=30$. This initial state may be experimentally relevant for cold atomic gases loaded on optical lattices [3]. To calculate the quantum dynamics we sample $10^{3}$ different initial conditions both for the coherent and for the Fock initial state, but in order to test convergence we went up to $10^{4}$ realizations. To quantify the overall relaxation of the density profile to its thermal configuration we introduce the dynamical distance

$$
d^{2}(t)=\frac{1}{L} \sum_{j=1}^{L}\left[\left\langle\hat{n}_{j}(t)\right\rangle-N\right]^{2} .
$$

Clearly, thermal equilibrium implies $d^{2}(t)=0$. In Fig. 1 we show the decay of $d^{2}(t)$ for both the coherent and the Fock initial states for different values of the coupling strength. In both cases for low coupling strength the system thermalizes quickly in a time scale of the order of one hopping [3,4]. As we increase the coupling strength a metastable state emerges in between the initial relaxation and the final decay to equilibrium. The lifetime of such a metastable state increases with the coupling strength until it becomes larger than the maximum time available in our simulation. For the Fock initial state the increase of the lifetime of the metastable state is more abrupt. In both cases, the density profile of the metastable state is closer to that of the initial state as we increase the coupling strength.

In Fig. 2 we show the relaxation of the kinetic energy $e_{\text {kin }}(t)=\operatorname{Tr}\left[\rho_{0} e^{i H t} H_{0} e^{-i H t}\right]$ for the coherent state initial condition for several values of the interaction strength. For the Fock initial state we obtain qualitatively the same results. We observe that for weak interactions $U / J \lesssim 0.1$ the kinetic energy prethermalizes in a time scale $t_{\mathrm{pt}}^{\text {weak }} \sim 10 J^{-1}$. For intermediate interaction strengths $0.2 \lesssim U / J \lesssim 0.7$ the kinetic energy also tends to a well-defined constant but with 

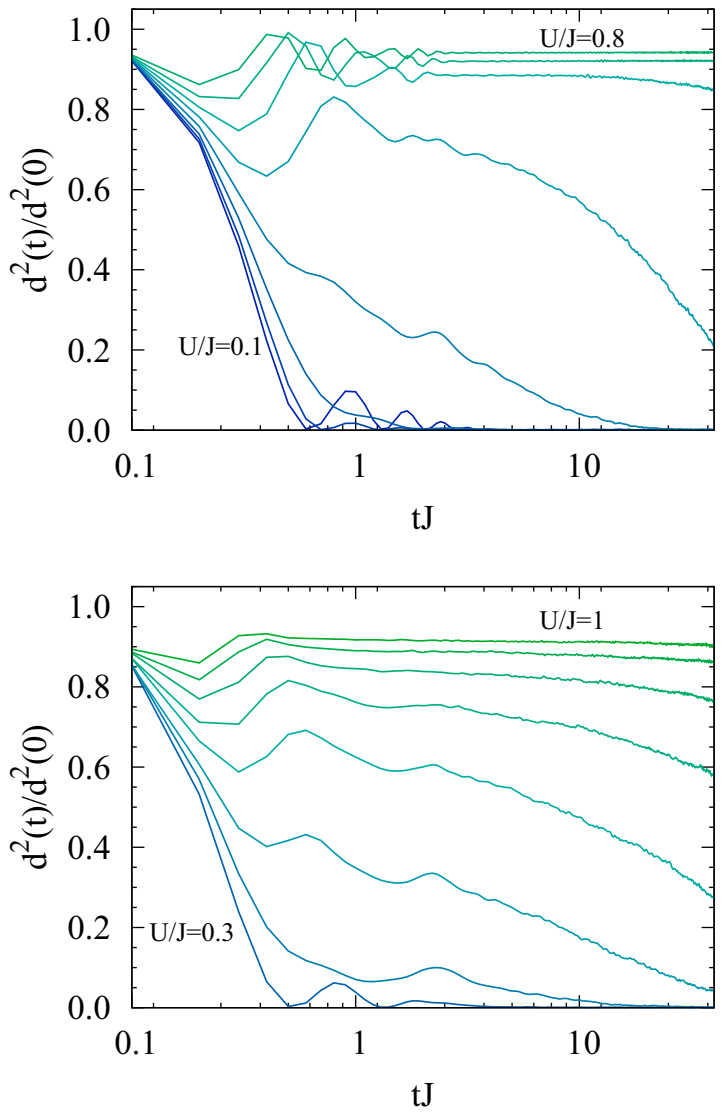

FIG. 1. (Color online) Dynamical distance $d(t)$ as a function of time for the Fock (upper) and coherent (lower) initial states. Different curves correspond to different interaction strengths $U / J$. Note the logarithmic scale on the time axis.

a larger relaxation time of the order of $t_{\mathrm{pt}}^{\mathrm{int}} \sim 30 \mathrm{~J}^{-1}$ where "int" stands for intermediate. Whereas for strong interaction strengths $U / J \gtrsim 0.7$ the prethermalization time scale is $t_{\mathrm{pt}}^{\text {strong }} \sim 3 J^{-1}$. The variety of prethermalization time scales can be understood from the fact that the quasifree modes behind the dephasing relaxation are qualitatively different

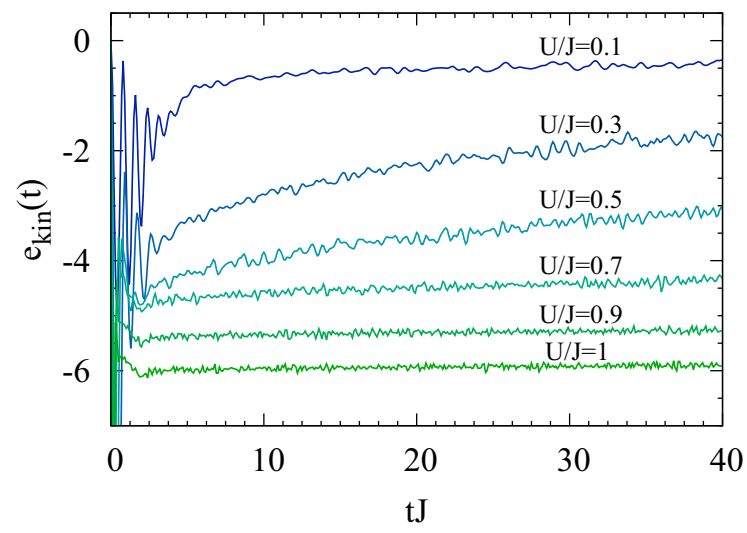

FIG. 2. (Color online) Relaxation of the kinetic energy for the coherent state initial condition. Different curves correspond to different interaction strengths $U / J$. Curves are vertically shifted for clarity.
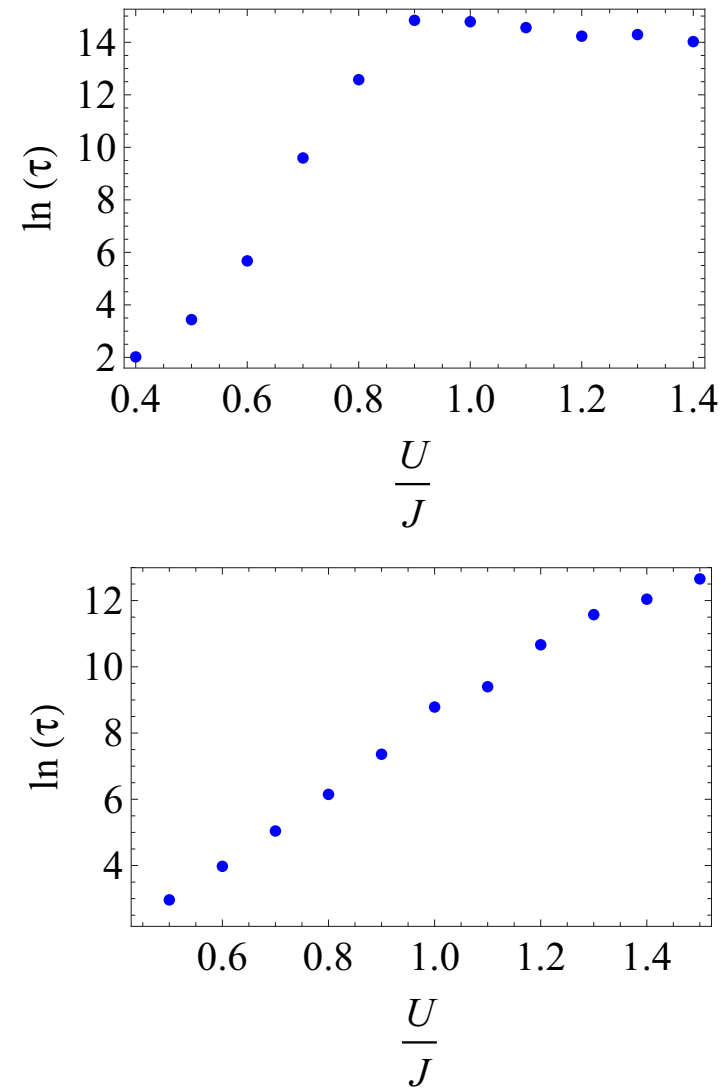

FIG. 3. (Color online) Logarithm of the relaxation time as a function of coupling $U / J$ for the Fock (upper) and coherent (lower) initial state.

for strong and weak interactions. For weak interactions, the quasifree modes are related to the momentum eigenmodes that diagonalize $H_{0}$ and are completely delocalized in space. For strong interactions, the quasifree modes are related to the eigenmodes that diagonalize $H_{\text {int }}$ and are completely localized excitations. For intermediate interactions there is a truly nontrivial regime for which the quasifree modes are neither completely localized nor delocalized. This suggests that the effective models that describe the short time dynamics in the three cases are completely different. However, a remarkable fact is that for strong interactions the prethermalization time is the same regardless the specific value of the coupling strength, while the relaxation time scale of the density profile shows large variations (see Fig. 3) due to the presence of metastable states.

The crossovers from fast thermalization to the metastable state dominated regime for the two different initial conditions occur in different coupling ranges. To obtain a decay time scale of $d^{2}(t)$ we fit an exponential to the tail of the decay. In Fig. 3 we show the results for the decay time scales for both initial conditions. We find that the decay time scales increase by several orders of magnitude as we increase the coupling strength in a small range. We find that the center of the crossover region is around $U / J \simeq 0.7$ for the Fock initial state and $U / J \simeq 1$ for the coherent state.

We should mention that while a detailed analysis of the dependence of the relaxation time scales on the system size is 
beyond the scope of the present work, preliminary results show that finite-size effects are almost negligible for the system size, time scales, and observables considered above.

The emergence of prethermalized states with such large lifetime may completely hinder the observation of the thermal equilibrium state of the system after the quench and certainly is at the root of the apparent lack of thermalization observed in earlier works $[19,21]$. We find the situation rather similar to that of glasses, systems that exhibit the typical two-step relaxation as a consequence of getting caught in extremely long-lived metastable states, whose lifetime can be astronomical for sufficiently low temperatures or high densities. In the next section we shall outline a more qualitative relation between the emergence of long-lived metastable states and glassiness by analyzing the properties of the mean-field trajectories associated with the quantum dynamics.

\section{GLASSY PROPERTIES IN MEAN-FIELD TRAJECTORIES}

In Ref. [29] it was proposed that the ability of a quantum system to thermalize is related to the ergodicity of the classical trajectories of the associated mean-field system, an idea that is also implicit in previous studies, such as Refs. [31,32]. In this section we shall show that the emergence of long-lived prethermalized states in the quantum dynamics of the Hubbard model is correlated with the lack of ergodicity of the classical trajectories that are used to build up the quantum dynamics.

To qualitatively introduce the discussion, in Fig. 4 we show a stroboscopic sampling of the position in phase space of the field $\psi_{1}$ for two different couplings. We can see that for small coupling $(U=0.5)$ the system explores the whole phase space while for large coupling $(U=1.5)$ it remains captured in a ring of finite width in the $\operatorname{Re}\left(\psi_{1}\right)$ vs $\operatorname{Im}\left(\psi_{1}\right)$ space. A similar behavior is observed when analyzing the behavior of the phasespace trajectory of the other sites. Going back to Fig. 1 we can check that indeed for $U / J=0.5$ the quantum density profile is thermalized for $t J=40$ while it is still trapped in the metastable state for $U / J=1.5$.

Additionally, we point out that the phase of the fields $\theta_{j}(t)=\arctan \left\{\operatorname{Im}\left[\psi_{j}(t)\right] / \operatorname{Re}\left[\psi_{j}(t)\right]\right\}$ turns out to be always ergodic, in the sense that along the dynamic evolution it visits all values between 0 and $2 \pi$, irrespective of the value of the coupling strength, for all trajectories. This fact is also illustrated by Fig. 4 where, even for the strong coupling regime, the phase of the field $\psi_{1}$ explores all values. The lack of ergodicity is thus encoded in the dynamics of the particle density at each site $n_{j}(t)=\left|\psi_{j}(t)\right|^{2}$.

We will now proceed to make a more detailed and quantitative analysis of the properties of the classical trajectories. To characterize them we introduce the mobility $K$ of each trajectory

$$
K[\psi(t)]=\Delta t \sum_{t=0}^{t_{\mathrm{bbs}}} \sum_{j=1}^{N}\left[\left|\psi_{i}(t+\Delta t)\right|^{2}-\left|\psi_{i}(t)\right|^{2}\right]^{2},
$$

where $t_{\mathrm{obs}}$ is the maximum observation time, i.e., the extension of the trajectories and $\Delta t$ is an UV cutoff that kills possible small oscillations leaving just the actual displacement in the phase space. $K[\psi(t)]$ is an extensive-in-time quantity that
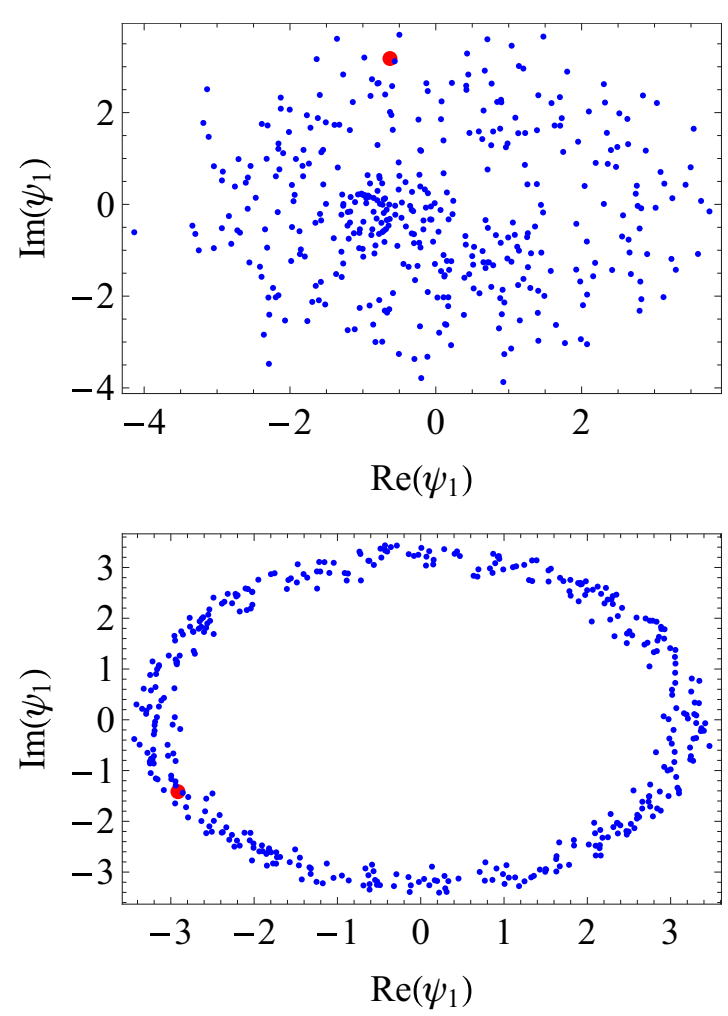

FIG. 4. (Color online) Position in the phase space $\operatorname{Re}\left(\psi_{1}\right)$ vs $\operatorname{Im}\left(\psi_{1}\right)$ for the first site of the chain observed at every $J \Delta t=0.1$ for $J t_{\mathrm{obs}}=40$ for $U / J=0.5$ (upper) and $U / J=1.5$ (lower). The big red dot corresponds to the initial configuration. Each site was chosen to have a random phase as the initial condition as the initial condition.

will typically be large for ergodic trajectories, while it will be generically small for nonergodic trajectories as can be intuitively seen from Fig. 4. Thus, $K[\psi(t)]$ can be used as an order parameter to distinguish ergodic (mobile) from nonergodic (immobile) trajectories.

Since we are interested in assessing the properties of the trajectories that are most relevant to the quantum motion we will introduce a measure on the space of classical trajectories given by the Wigner function of the quantum initial state that we wish to consider. In the case of the coherent state, the measure $p_{C}\left(\psi_{0}, \psi_{0}^{*}\right)$ is well defined while in the case of the Mott insulating state we can choose any well-defined distribution that approximates $p_{F}\left(\psi_{0}, \psi_{0}^{*}\right)$, such as $p_{\delta}$ or $p_{g}$. For simplicity, we shall restrict the following analysis to the coherent state case.

In Fig. 5 we show the mobility histogram from an ensemble of $2 \times 10^{3}$ trajectories sampled according to $p_{C}\left(\psi_{0}, \psi_{0}^{*}\right)$ for four different values of $U / J$. The center of the distributions monotonically shifts to lower values of the mobility as we increase $U / J$, but the dispersion around the center decreases for large coupling constants. In particular, for sufficiently large coupling all the trajectories have a very small mobility with a small dispersion, showing that all trajectories are freezed, at least up to time scales of the order of $t_{\mathrm{obs}}$. However, there is an intermediate coupling regime $0.3 \lesssim U / J \lesssim 0.6$, which corresponds to the intermediate regime described when analyzing the relaxation of the kinetic energy in the previous 


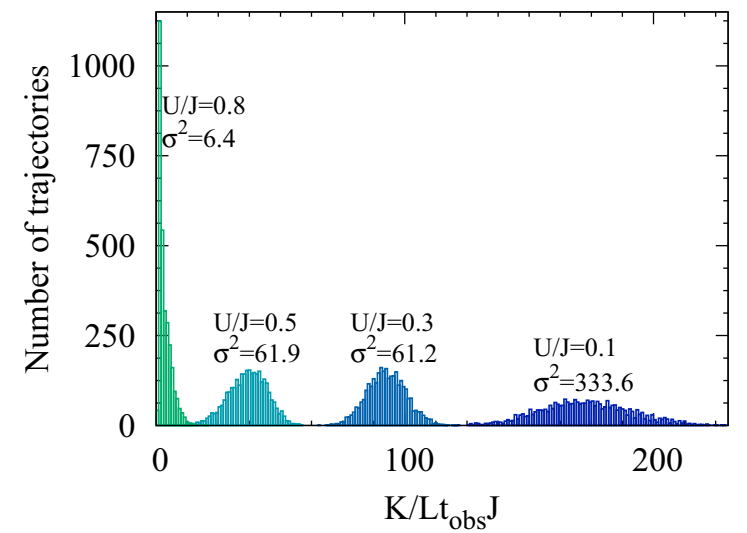

FIG. 5. (Color online) Histogram of the mobility $K[\psi(t)]$ for ensembles of $2 \times 10^{3}$ trajectories sampled according to the Wigner function of the coherent state initial condition $p_{C}\left(\psi_{0}, \psi_{0}^{*}\right)$ for four different values of $U / J$.

section, for which the dispersion almost does not change. This confirms that inactive, nonergodic classical trajectories are related to the existence of nonthermal metastable states in the quantum dynamics. Moreover, since the shift of the center of the histograms is continuous we may expect that for intermediate couplings there should be a coexistence of active and inactive trajectories, in which case the mobility of the trajectories would depend on the details of the initial conditions. This is indeed the case. This observation can be cast in the language of a dynamical phase transition $[35,37-39,45]$ taking place in the ensemble of classical trajectories.

A dynamical phase transition can be defined in complete analogy with equilibrium phase transitions [39]. In the statistical mechanics description of an equilibrium phase transition, one often considers an ensemble of microstates of a system and an extensive-in-size quantity that characterizes each microstate of the system, such as the energy of each configuration in a classical Ising model. Then one introduces a canonical probability distribution that gives a different weight to each configuration, depending on the value that the extensive parameter takes for each configuration. If the extensive quantity that we choose is energy, the canonical probability distribution is the familiar $\frac{1}{Z} e^{-\beta H}$, where $H$ is the Hamiltonian; $\beta$, the intensive conjugate field of the energy, is the inverse temperature; and $Z$ is the partition function. To analyze a phase transition we consider the average of a given order parameter, like the magnetization in the case of the Ising model, among all microstates weighted with the canonical probability distribution. We may observe the phase transition as an abrupt change in the value of the weighted average of the order parameter as a function of the intensive parameter $\beta$. Then we talk about a temperature-driven phase transition in the ensemble of microstates. For the dynamical phase transition we make statistical mechanics with the trajectories of the system and consider them as the "microstates." In the present case, we choose the mobility $K[\psi(t)]$ as an extensive-in-time quantity that characterizes each trajectory. We then construct a canonical probability distribution defined over the ensemble of trajectories coupling the extensive-in-time quantity $K[\psi(t)]$ with an intensive field $s$ which is analogous to temperature in
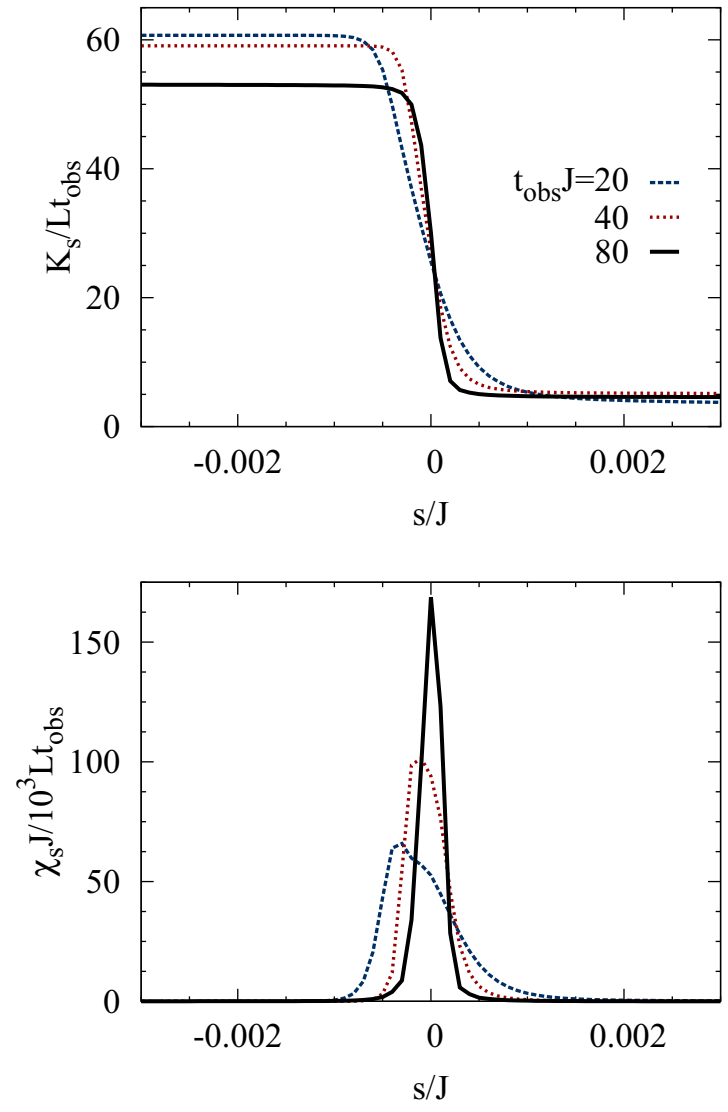

FIG. 6. (Color online) Dynamical phase transition in trajectory space. Upper panel: Averaged order parameter $K_{s}$ as a function of the intensive field $s$ for an ensemble of $10^{3}$ trajectories sampled according to the coherent state Wigner function for $U / J=0.53$. Lower panel: Dynamical susceptibility $\chi_{s}$.

the example discussed before. This distribution will be

$$
P_{s}[\psi(t)]=\frac{1}{Z_{s}} P_{0}[\psi(t)] e^{-s K[\psi(t)]},
$$

where $P_{0}[\psi(t)]$ is the $s=0$ probability distribution that we take as the Wigner function of the coherent state $p_{C}\left(\psi_{0}, \psi_{0}^{*}\right)$ as discussed earlier and $Z_{s}$ is the partition function

$$
Z_{s}=\sum_{\psi(t)} P_{s}[\psi(t)]
$$

We will compute expectation values in the ensemble of trajectories by summing over all trajectories weighted with $P_{s}[\psi(t)]$ in the following fashion:

$$
\Omega_{s}=\langle\Omega[\psi(t)]\rangle_{s}=\frac{1}{Z_{s}} \sum_{\psi(t)} P_{s}[\psi(t)] \Omega[\psi(t)],
$$

where $\Omega[\psi(t)]$ is a trajectory functional. This way of averaging will give a different weight to mobile and immobile trajectories depending on the value of $s$ : for larger $s$ more relative weight is assigned to inactive trajectories and viceversa.

To analyze the phase transition we take the mobility $K[\psi(t)]$ as the order parameter. In Fig. 6 we show the average order parameter $K_{s}$ as a function of $s$ for $U / J=0.53$, a value of $U / J$ for which we have observed coexistence of 


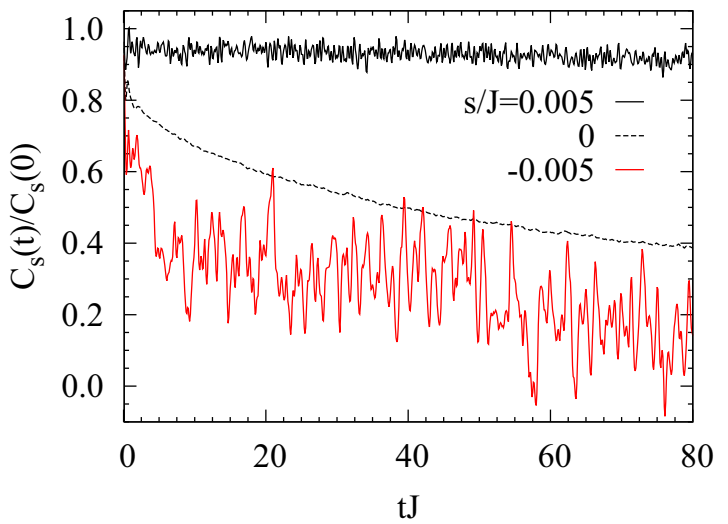

FIG. 7. (Color online) Correlation function $C_{s}(t)$ as a function of time for $U / J=0.53$ in the active $(s<0)$ and inactive $(s>0)$ phases. We also show, for comparison, the correlation function at coexistence $(s=0)$.

active and inactive trajectories. The behavior of $K_{s}$ mimics the behavior of the order parameter in a finite-volume equilibrium phase transition [39]: it shows a marked step between two well-defined values corresponding to the two different phases in the ensemble (active and inactive phases) and, moreover, while increasing $t_{\mathrm{obs}}$ (analogous to the volume in equilibrium) the step in $K_{s}$ becomes more and more sharp. This can be appreciated more clearly looking at the susceptibility:

$$
\chi_{s}=-\frac{\partial K_{s}}{\partial s}=\left\langle\left(K[\psi(t)]-K_{s}\right)^{2}\right\rangle_{s}
$$

which exhibits a peak that grows while increasing $t_{\mathrm{obs}}$. A similar scaling behavior can be observed with increasing $L$ [39]. The critical value is located around zero, $s^{*}=0$. This dynamical phase transition is very similar to that found first in kinetically constrained models of glasses [37] and then in atomistic models of glass formers [39] and quantum systems [45]. The main difference that we observe with respect to the works $[37,39,45]$ is that the present dynamic phase transition does not seems to be of first order. In a first-order (dynamic or static) phase transition the distribution of the order parameter at coexistence is bimodal due to surface tension effects between the coexisting domains of different phases. In the case analyzed in this work the order parameter distribution at coexistence ( $s \simeq 0$ for $U / J=0.5$ ) is unimodal, as can be inferred looking at Fig. 5, which is compatible with a continuous phase transition.

To better characterize the physical properties of the phases we will discuss an ergodicity measure. We define the overlap correlation function

$$
C_{s}(t)=\sum_{j=1}^{L}\left\langle\left(n_{j}(t)-N\right)\left(n_{j}(0)-N\right)\right\rangle_{s} .
$$

$C_{s}(t)$ quantifies the overlap between the configuration at time $t$ and the initial density configuration. The extent to which it is nonzero in the limit of large $t$ is a measure of nonergodicity. In Fig. 7 we show the correlation function $C_{s}(t)$ for the active (ergodic) and inactive (nonergodic) phases. In the active phase $(s<0)$ the correlation function rapidly relaxes to a small
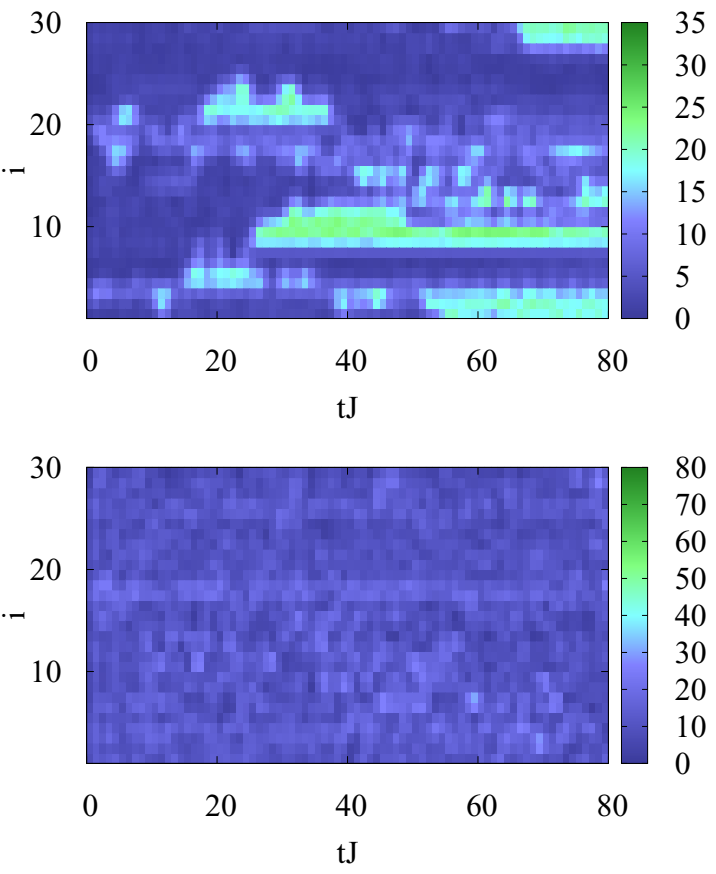

FIG. 8. (Color online) False color plot of the local mobility $Q_{i}(t)$ for two representative trajectories corresponding to $U / J=0.6$ (upper) and $U / J=0.1$ (lower).

value, while for the inactive phase $(s>0)$ trajectories remain trapped in a single state throughout the observation time.

In classical models of glasses such a dynamical phase transition represents one of the main theories to account for a striking and distinctive feature of glassy materials: dynamic heterogeneity [35]. In contrast with normal fluids, the relaxation of glasses is heterogeneous in space: fast and slow regions are clustered together. We found that individual mean-field trajectories (that correspond to single solutions of the Gross-Pitaevskii equation) exhibit the phenomenon of dynamical heterogeneity. We shall define a local measure of mobility $Q_{i}(t)$ as

$$
Q_{i}(t)=\left|n_{i}(t)-n_{i}(0)\right|,
$$

which measures how much the in-site density differs from the initial value. In Fig. 8 we show density plots of $Q_{i}(t)$ for representative trajectories corresponding to $U / J=0.1$ and 0.6 . For $U / J=0.1$ (the weak coupling regime) the dynamics of the system, as captured by the indicator $Q_{i}(t)$, is fairly homogeneous. For $U / J=0.6$ (entering the strong coupling regime), sites with high local mobility and low local mobility are clustered together in space-time. This is the signature of dynamic heterogeneity. It is remarkable that the simple Gross-Pitaevskii equation is able to generate such complicated dynamics.

The relation between the dynamical phase transition and the dynamical heterogeneity can be explained using simple reasoning: the dynamical phase transition picture implies that for intermediate values of $U / J$ different initial conditions may trigger slow or fast dynamics, depending only on the details of the initial condition; if we consider a sufficiently large system with short-range interactions, distant regions in space will not be correlated and if one region has a "fast" initial 
condition and the other a "slow" initial condition, dynamical heterogeneity may arise. Moreover, by the same argument, during the dynamical process slow regions may turn into fast regions and vice versa. Contrary to what is observed in Refs. [46,47], the quantum dynamics does not show dynamical heterogeneity. The average needed to obtain the quantum dynamics from the individual classical trajectories washes out any initial condition dependent dynamical heterogeneous pattern, which is associated with the fact that the density profile has a well-defined relaxation time scale as seen before.

\section{CONCLUSIONS}

We have studied the dynamics of the Bose-Hubbard model following a quantum quench from an inhomogeneous initial state. Using the TWA we have analyzed the dynamics of the density profile of the system, which exhibits long-lived prethermalization plateaus for moderate and large couplings. We were able to relate this fact to the nonergodic properties of the associated mean-field system. In particular, we have shown that the ensemble of mean-field trajectories that are relevant to the quantum motion undergoes a dynamic phase transition from an active to an inactive phase, much like what is found in classical models of glasses. We have also shown that this transition is related to the phenomenon of dynamic heterogeneity in the classical trajectories, one of the hallmarks of glassiness. In sum, we believe that our work opens a new perspective on the dynamics of closed quantum systems by relating the physics of classical glasses to the well-known phenomenon of prethermalization.

As a direction for future work, we think that to disentangle finite-size effects from prethermalization effects in the determination of the thermalization time scales is of primary importance. This goal is beyond the capabilities of standard numerical techniques (which typically can deal either with large systems and short times or with small systems and arbitrary times) but may be within reach for the TWA.

\section{ACKNOWLEDGMENTS}

We acknowledge very useful discussions with Andrea Gambassi and Pablo R. Ponte. We are grateful to Marcos Rigol for a correspondence exchange about the finite-size issue. I.S.L. thanks ICTP where part of this project was done. This work was partially supported by CONICET (PIP 0662), ANPCyT (PICT 2010-1907), and UNLP (PID X497), Argentina.
[1] A. Polkovnikov, K. Sengupta, A. Silva, and M. Vengalattore, Rev. Mod. Phys. 83, 863 (2011).

[2] I. Bloch, J. Dalibard, and W. Zwerger, Rev. Mod. Phys. 80, 885 (2008).

[3] S. Trotzky, Y.-A. Chen, A. Flesch, I. P. McCulloch, U. Schollwck, J. Eisert, and I. Bloch, Nat. Phys. 8, 325 (2012).

[4] S. Sorg, L. Vidmar, L. Pollet, and F. Heidrich-Meisner, Phys. Rev. A 90, 033606 (2014).

[5] M. Rigol, V. Dunjko, and M. Olshanii, Nature (London) 452, 854 (2008).

[6] M. Rigol, Phys. Rev. Lett. 112, 170601 (2014).

[7] T. Kinoshita, T. Wenger, and D. S. Weiss, Nature (London) 440, 900 (2006).

[8] J. Berges, S. Borsányi, and C. Wetterich, Phys. Rev. Lett. 93, 142002 (2004).

[9] M. Moeckel and S. Kehrein, Phys. Rev. Lett. 100, 175702 (2008).

[10] M. Eckstein, M. Kollar, and P. Werner, Phys. Rev. Lett. 103, 056403 (2009).

[11] M. Kollar, F. A. Wolf, and M. Eckstein, Phys. Rev. B 84, 054304 (2011).

[12] N. Nessi, A. Iucci, and M. A. Cazalilla, Phys. Rev. Lett. 113, 210402 (2014).

[13] M. Gring, M. Kuhnert, T. Langen, T. Kitagawa, B. Rauer, M. Schreitl, I. Mazets, D. A. Smith, E. Demler, and J. Schmiedmayer, Science 337, 1318 (2012).

[14] F. H. L. Essler, S. Kehrein, S. R. Manmana, and N. J. Robinson, Phys. Rev. B 89, 165104 (2014).

[15] M. Marcuzzi, J. Marino, A. Gambassi, and A. Silva, Phys. Rev. Lett. 111, 197203 (2013).

[16] M. Stark and M. Kollar, arXiv:1308.1610.
[17] T. Langen, S. Erne, R. Geiger, B. Rauer, T. Schweigler, M. Kuhnert, W. Rohringer, I. E. Mazets, T. Gasenzer, and J. Schmiedmayer, Science 348, 207 (2015).

[18] M. Rigol, Phys. Rev. Lett. 103, 100403 (2009).

[19] C. Kollath, A. M. Läuchli, and E. Altman, Phys. Rev. Lett. 98, 180601 (2007).

[20] G. Roux, Phys. Rev. A 79, 021608 (2009).

[21] G. Carleo, F. Becca, M. Schiró, and M. Fabrizio, Sci. Rep. 2, 243 (2012).

[22] M. P. Kennett and D. Dalidovich, Phys. Rev. A 84, 033620 (2011).

[23] M. A. Cazalilla, R. Citro, T. Giamarchi, E. Orignac, and M. Rigol, Rev. Mod. Phys. 83, 1405 (2011).

[24] M. Kennett, ISRN Cond. Matter Phys. 2013, 393616 (2013).

[25] S. S. Natu, K. R. A. Hazzard, and E. J. Mueller, Phys. Rev. Lett. 106, 125301 (2011).

[26] C.-L. Hung, X. Zhang, N. Gemelke, and C. Chin, Phys. Rev. Lett. 104, 160403 (2010).

[27] D. McKay, U. Ray, S. Natu, P. Russ, D. Ceperley, and B. DeMarco, Phys. Rev. A 91, 023625 (2015).

[28] M. Srednicki, Phys. Rev. E 50, 888 (1994).

[29] J. G. Cosme and O. Fialko, Phys. Rev. A 90, 053602 (2014).

[30] A. Polkovnikov, Ann. Phys. (NY) 325, 1790 (2010).

[31] G. Jona-Lasinio and C. Presilla, Phys. Rev. Lett. 77, 4322 (1996).

[32] A. Cassidy, D. Mason, V. Dunjko, and M. Olshanii, Phys. Rev. Lett. 102, 025302 (2009).

[33] T. Castellani and A. Cavagna, J. Stat. Mech.: Theor. Exp. (2005) P05012.

[34] A. Cavagna, Phys. Rep. 476, 51 (2009).

[35] D. Chandler and J. P. Garrahan, Annu. Rev. Phys. Chem. 61, 191 (2010). 
[36] L. Berthier, G. Biroli, J.-P. Bouchaud, L. Cipelletti, and W. van Sarloos, Dynamical Heterogeneities in Glasses, Colloids and Granular Media (Oxford Science Publications, Oxford, 2011).

[37] J. P. Garrahan, R. L. Jack, V. Lecomte, E. Pitard, K. van Duijvendijk, and F. van Wijland, Phys. Rev. Lett. 98, 195702 (2007).

[38] J. P. Garrahan, R. L. Jack, V. Lecomte, E. Pitard, K. van Duijvendijk, and F. van Wijland, J. Phys. A: Math. Theor. 42, 075007 (2009).

[39] L. O. Hedges, R. L. Jack, J. P. Garrahan, and D. Chandler, Science 323, 1309 (2009).
[40] A. Polkovnikov, S. Sachdev, and S. M. Girvin, Phys. Rev. A 66, 053607 (2002).

[41] P. Blakie, A. Bradley, M. Davis, R. Ballagh, and C. Gardiner, Adv. Phys. 57, 363 (2008).

[42] A. Sinatra, C. Lobo, and Y. Castin, J. Phys. B 35, 3599 (2002).

[43] M. K. Olsen and A. S. Bradley, Opt. Commun. 282, 3924 (2009).

[44] A. Polkovnikov, Phys. Rev. A 68, 033609 (2003).

[45] J. P. Garrahan and I. Lesanovsky, Phys. Rev. Lett. 104, 160601 (2010).

[46] Z. Nussinov, P. Johnson, M. J. Graf, and A. V. Balatsky, Phys. Rev. B 87, 184202 (2013).

[47] Z. Nussinov, arXiv:1203.4648. 